\title{
Laminar-turbulent cycles in inclined lock-exchange flows
}

\author{
Yukie Tanino, ${ }^{*}$ Frédéric Moisy, $^{\dagger}$ and Jean-Pierre Hulin ${ }^{\ddagger}$ \\ Université Pierre et Marie Curie-Paris 6, Université Paris-Sud, CNRS, \\ Laboratoire FAST, Bât. 502, Campus Universitaire, Orsay F-91405, France
}

(Received 13 August 2011; revised manuscript received 16 February 2012; published 11 June 2012)

\begin{abstract}
We consider strongly confined, stably stratified shear flows generated as a lock exchange in a tube inclined at an angle of $\theta=45^{\circ}$. This paper focuses on a transitional regime, in which the flow alternates between two distinct states: laminar, parallel shear flow and intense transverse motion characteristic of turbulence. Laminar-turbulent cycles were captured at Atwood numbers At $\equiv\left(\rho_{2}-\rho_{1}\right) /\left(\rho_{1}+\rho_{2}\right)$ ranging from $2.45 \times 10^{-3}$ to $4.0 \times 10^{-3}$, where $\left(\rho_{1}, \rho_{2}\right)$ are the initial densities of the two fluids, with multiple cycles observed at At $=2.55 \times 10^{-3}$. The evolution of the density and velocity fields in these flows was measured simultaneously using laser-induced fluorescence and particle image velocimetry. During each laminar-turbulent cycle, the axial velocity exhibits a distinctive ramp-cliff pattern, indicating that the flow accelerates as it relaminarizes, then decelerates rapidly as the Kelvin-Helmholtz billows break down. Within the range of experimental conditions, transverse stratification does not directly determine the onset of instability. Instead, the data suggest that a necessary criterion for the onset of instability is for the local Reynolds number to exceed 2200, with only a weak dependence on the Richardson number.
\end{abstract}

DOI: 10.1103/PhysRevE.85.066308

PACS number(s): 47.20.Ft, 47.27.Cn, 47.20.Bp

\section{INTRODUCTION}

Stably stratified shear flows are ubiquitous in the environment, as evidenced by observations in the atmosphere [1-5], in oceans [6-8], in estuaries [9,10], and in lakes [11-13]. In such flows, stratification and viscosity tend to stabilize the flow [14], while the shear tends to destabilize it via Kelvin-Helmholtz $(\mathrm{KH})$ instability. Indeed, $\mathrm{KH}$ billows are well documented in the atmosphere $[1,2,5]$ and are regarded as the primary precursor for turbulence in calm weather.

The stability of these flows is generally governed by the gradient Richardson number and the Reynolds number. The Richardson number characterizes the competition between shear and stratification, while the Reynolds number characterizes that between inertia and viscosity. Physical reasoning suggests that there is a critical Reynolds number below which the flow is stabilized by viscosity for all Richardson numbers and, conversely, that there is a critical Richardson number above which the flow is stabilized by buoyancy for all Reynolds numbers [5,6,15]. Indeed, this framework has been verified theoretically for stratified viscous shear flows confined between parallel boundaries - a configuration similar to that considered in the present paper [16,17].

However, while the theory may provide a necessary criterion for instability, the conditions under which the instability first becomes apparent in an experiment have been found to deviate significantly. For example, Defina et al. [17] found the onset of instability in flows generated by the classic Thorpe-type tilting tank to occur over a wide range of Richardson numbers and Reynolds numbers, with a Reynolds number dependence opposite to that predicted by their stability analysis. In this paper, we will also see that a single value of

\footnotetext{
*ytanino@alum.mit.edu; currently in the Department of Earth Science and Engineering, Imperial College London.

${ }^{\dagger}$ moisy@ fast.u-psud.fr

${ }^{\ddagger}$ hulin@fast.u-psud.fr
}

the Richardson number does not distinguish flows that subsequently exhibit turbulence from those that do not. More generally, quantitative descriptions of the evolution of the velocity field and stratification in stratified shear flows are limited, with existing literature focusing largely on conditions approaching the onset of instability in simple, steady basic flows.

In the present study, we document quantitatively the evolution of stratified shear flows that exhibit two distinct states: near-parallel, laminar flow, and brief periods of rapid fluctuations reminiscent of turbulence. We will refer to the sequence of relaminarization, the eventual onset of instability, and the subsequent breakdown of the parallel shear as a laminar-turbulent cycle. Emphasis will be placed in a flow that alternates repeatedly between the two states, reestablishing the same magnitude of stratification and shear in each successive cycle.

We consider specifically flows under strong confinement, generated as a lock exchange in an inclined tube. Here, a long tube is divided in half by a removable transverse partition. The upper and lower halves of the tube are filled with denser and lighter fluids, respectively. When the partition is removed, the axial density gradient initiates an exchange flow, with the heavier fluid propagating downward along the lower portion of the tube cross section and the lighter fluid propagating along the upper portion in the opposite direction. Unlike the classic horizontal configuration (e.g., Ref. [18]), the inclined lock exchange is driven by the axial component of gravity, while the transverse component contributes primarily to segregation [19].

We emphasize that this paper is focused on the evolution of the stratified shear flow that is established far downstream of the propagating fronts, and not on the propagation speed of the fronts or the profile of the interface. Such flows exhibit three distinct regimes, which may be obtained by changing the angle of incline to the vertical $(\theta)$ and the Atwood number At $\equiv$ $\left(\rho_{2}-\rho_{1}\right) /\left(\rho_{1}+\rho_{2}\right)$, where $\rho_{1}$ and $\rho_{2}\left(>\rho_{1}\right)$ are the densities of the two fluids prior to flow initiation [20,21]. At small $\theta \lesssim 30^{\circ}$ and large $\mathrm{At} \gtrsim O\left(4 \times 10^{-3}\right)$, the flow is uniformly turbulent; 
i.e., it does not exhibit slow variations in time or in $x$. At large $\theta\left(>45^{\circ}\right)$ and small $\operatorname{At}\left(\leqslant 10^{-3}\right)$, the flow is permanently laminar. Between these limits is the third regime, which is associated with sustained laminar-turbulent alternation [20-22].

In this paper, laboratory measurements of the density and velocity fields in lock-exchange flows in a tube inclined at $\theta=45^{\circ}$ at At ranging from $1.30 \times 10^{-3}$ to $1.19 \times 10^{-2}$ are presented. These values of $(\theta, \mathrm{At})$ overlap with the transition from laminar flow to uniform turbulence. Spatiotemporal diagrams of the streamwise velocity and root-mean-square (rms) value of the transverse velocity are presented, and the observed dependence on At is discussed (Sec. III A). The evolution of the streamwise velocity, the rms of the transverse velocity, and the transverse density gradient in and between consecutive laminar-turbulent cycles are described (Sec. III B). Finally, the relevance of the Richardson and Reynolds numbers as criteria for the onset of instability is explored (Sec. III C).

\section{MATERIALS AND METHODS}

Lock-exchange flows were generated in a $d=20 \mathrm{~mm}$ diameter Plexiglas tube consisting of two $1670 \mathrm{~mm}$ sections separated by a transverse partition that can be opened or closed. The tube was oriented at $\theta=45^{\circ}$ to the vertical for all runs considered in the present paper. The Cartesian coordinates $(x, y, z)$ are defined as illustrated in Fig. 1. The $x$ axis is aligned with the tube axis, with the origin defined at the partition.

Prior to each experiment, the partition was closed. The lower tube was filled with an aqueous solution of rhodamine $6 \mathrm{G}$ of concentration $c_{1}=(161 \pm 2) \times 10^{-3} \mathrm{~g} \mathrm{~m}^{-3}$ and density $\rho_{1}=999 \mathrm{~kg} \mathrm{~m}^{-3}$. The upper tube was filled with an aqueous solution of calcium chloride and rhodamine $6 \mathrm{G}$ of density $\rho_{2}\left(>\rho_{1}\right)$ and rhodamine concentration $c_{2}$ of either $(201 \pm 3) \times 10^{-4} \mathrm{~g} \mathrm{~m}^{-3}$ or $(215 \pm 3) \times 10^{-4} \mathrm{~g} \mathrm{~m}^{-3}$. The density and temperature of the two fluids were measured using a digital density meter (Anton Paar DMA35N, $\pm 1 \mathrm{~kg} \mathrm{~m}^{-3}$, $\pm 0.2^{\circ} \mathrm{C}$ ); the temperatures of the two fluids were equal within the accuracy of the equipment in all experiments considered. Both liquids were seeded with glass spheres (Sphericel ${ }^{\circledR}$ 110P8) for particle image velocimetry (PIV).

An experiment began with the removal of the partition at $t=0$. The density gradient generated an exchange flow,

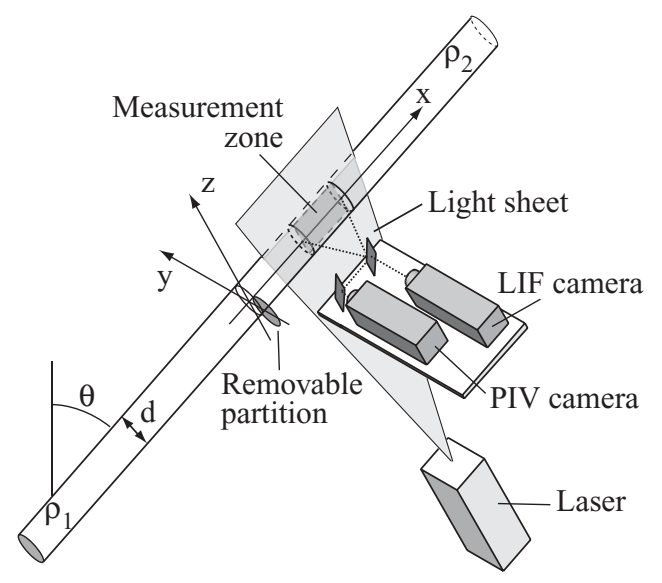

FIG. 1. Central section of the experimental setup. Not to scale. whereby the denser fluid propagated toward the bottom tube preferentially along the lower section of the tube wall $(z<0)$, and the lighter fluid propagated in the opposite direction along the upper section $(z>0)$. Between the leading fronts of the two currents, the fluid was stably stratified.

As the exchange flow propagated, a pulsed laser sheet of wave length $\lambda=532 \mathrm{~nm}$ (Continuum MINILITE-PIV, Electro-Optics, Inc.) illuminated the vertical $x-z$ plane passing through the axis of the tube (Fig. 1). In this plane, the tube walls are at $\tilde{z} \equiv z / d= \pm 1 / 2$. In this paper, ${ }^{\sim}$ denotes a nondimensionalized parameter; the characteristic length and density used for normalization are $d$ and $\rho_{2}-\rho_{1}$, respectively. The laser sheet was approximately $2 \mathrm{~mm}$ thick. To reduce optical distortion from the cylindrical geometry, a large section of the upper tube was encased in a transparent, square cell oriented so that two sides were parallel with the laser plane. The space between the upper tube and the square cell was filled with water. A long-pass dichroic mirror was used to separate the laser-induced fluorescence (LIF) of rhodamine 6G (peak $\lambda=555 \mathrm{~nm}$ ) from the incoming laser light and the reflection of the light by the seeding material $(\lambda=532 \mathrm{~nm})$. One CCD camera (Roper Scientific, 1392 pix $\times 1040$ pix) captured the LIF of rhodamine $6 \mathrm{G}$, and a second CCD camera (LaVision Flowmaster, 1280 pix $\times 1024$ pix) imaged the seeding particles. The two cameras were positioned so that their fields of view coincided to within 3 pix within the $65 \times 20 \mathrm{~mm}$ rectangular region of interest centered between $x=323 \mathrm{~mm}$ and $x=360 \mathrm{~mm}$ from the partition. The resolution of the captured images varied between 19.0 and 19.4 pix $\mathrm{mm}^{-1}$ across different runs. The two cameras captured images simultaneously at 3.9 frames per second. The experiment was terminated when disturbances associated with the arrival of the fronts at the ends of the tube reached the imaging region.

From the images of seeding particles, the local longitudinal $(x)$ and transverse $(z)$ velocity components, $u$ and $w$, were determined by PIV using the software DaVis (LaVision GmbH). From the images of LIF, the instantaneous concentration of rhodamine $6 \mathrm{G}, c(x, z, t)$, was determined using a third-order polynomial fitted to the recorded fluorescence intensity for five known concentrations in the range $c_{2} \leqslant c \leqslant c_{1}$. The density, $\rho(x, z, t)$, is related to rhodamine concentration by

$$
\frac{\rho(x, z, t)-\rho_{1}}{\rho_{2}-\rho_{1}}=\frac{c_{1}-c(x, z, t)}{c_{1}-c_{2}} .
$$

Six experimental runs, with At ranging from $1.30 \times 10^{-3}$ to $1.19 \times 10^{-2}$, are considered in the present paper (Table I). The Schmidt number remained approximately constant at $\mathrm{Sc}=v / D_{m} \approx 3400-3500$, where the molecular diffusion coefficient is taken to be $D_{m}=3 \times 10^{-6} \mathrm{~cm}^{2} \mathrm{~s}^{-1}$ (Ref. [24], Fig. 18.10), and the kinematic viscosity $v$ is interpolated from values for aqueous calcium chloride reported in Ref. [25]. The corresponding global Reynolds number, $\operatorname{Re}_{t}=2 V_{t} d / \nu$, defined using the inertial velocity scale $V_{t}=\sqrt{g \cos \theta \text { Atd }}$, where $g$ is gravitational acceleration, ranges from $\operatorname{Re}_{t}=530$ to 1540 . Alternative combinations of variables may also be used to characterize the flow; the Rayleigh number, $\mathrm{Ra}=$ $2 \operatorname{Atg} d^{3} /\left(\nu D_{m}\right)$, may be of particular interest. However, Ra can be written in terms of the parameters defined above as $\mathrm{Ra} \equiv \mathrm{Sc}_{\mathrm{Re}}^{2} /(2 \cos \theta)$. 
TABLE I. Experimental conditions. $\tau_{N}$ is the period corresponding to the global Brunt-Väisälä frequency [Eq. (4)]. $V_{f}$ is the equilibrium (long-time) front velocity as interpolated from measurements reported in Refs. [20] (runs A-D) and [23] (runs E, F).

\begin{tabular}{lccccc}
\hline \hline Run & At & $\begin{array}{c}\rho_{1} \\
{\left[\mathrm{~kg} \mathrm{~m}^{-3}\right]}\end{array}$ & $\begin{array}{c}\rho_{2} \\
{\left[\mathrm{~kg} \mathrm{~m}^{-3}\right]}\end{array}$ & $\operatorname{Re}_{t}$ & $\begin{array}{c}V_{f} \\
{\left[\mathrm{~cm} \mathrm{~s}^{-1}\right]}\end{array}$ \\
\hline A & $1.30 \times 10^{-3}$ & 998.9 & 1001.5 & 530 & 6.6 \\
B & $2.45 \times 10^{-3}$ & 999.0 & 1003.9 & 730 & 4.8 \\
C & $2.55 \times 10^{-3}$ & 998.8 & 1003.9 & 740 & 4.7 \\
D & $4.0 \times 10^{-3}$ & 998.6 & 1006.6 & 920 & 3.8 \\
E & $1.19 \times 10^{-2}$ & 998.8 & 1022.9 & 1540 & 2.2 \\
F & $1.19 \times 10^{-2}$ & 998.9 & 1023.0 & 1540 & 2.2 \\
\hline \hline
\end{tabular}

\section{EXPERIMENTAL RESULTS AND DISCUSSION}

The dominant temporal and spatial scales in flows that alternate between laminar and turbulent states are those of turbulent fluctuations and those associated with the laminarturbulent alternation. In this paper, quantitative analyses are in terms of properties averaged over the streamwise span of the imaging region, denoted by \langle\rangle$_{x}$. Further, these variables are temporally averaged over $t \pm 0.5 \mathrm{~s}$. These averaging windows are much smaller than the characteristic scales of laminar-turbulent alternation and longer than those of the turbulent fluctuations.

As will be shown in Sec. IIIC, $u$ undergoes large, slow oscillations during relaminarization. These oscillations preclude the Reynolds decomposition of $u$ and, consequently, the calculation of the turbulent kinetic energy. Instead, we take advantage of the low values of the temporal average of the transverse velocity and use its rms, $\left\langle w^{2}\right\rangle_{x}^{1 / 2}(z, t)$, as a proxy for the strength of turbulence.

\section{A. The transition between laminar, intermittent turbulence, and uniform turbulence regimes}

We first examine the basic features of the flow at different At. Figure 2 presents, at four At, spatiotemporal diagrams of $u$ at the middle of the imaging region and $\left\langle w^{2}\right\rangle_{x}^{1 / 2}$. At the smallest At $=1.30 \times 10^{-3}$, only slow, weak variations can be seen in $u$, with the exception of the passage of the front and a weak disturbance at $t=90-100 \mathrm{~s}$. Similarly, $\left\langle w^{2}\right\rangle_{x}^{1 / 2}$ remained near zero, indicating laminar flow [Fig. 2(a) and 2(e)].

At At $=2.55 \times 10^{-3}$, extended periods of low $\left\langle w^{2}\right\rangle_{x}^{1 / 2}$ and predominantly parallel shear flow were interspersed by distinct, brief periods of large $\left\langle w^{2}\right\rangle_{x}^{1 / 2}$ and small $u$ [Fig. 2(b) and 2(f)]. These two states are interpreted as relaminarized flow and turbulent bursts, respectively. The alternation between the two states was regular, and three relaminarization-turbulent cycles can be readily identified [Fig. 2(f)].

At At $=4.0 \times 10^{-3}$, distinct periods of low $\left\langle w^{2}\right\rangle_{x}^{1 / 2}$ can still be identified, but they were brief and infrequent [Fig. 2(g)]. Similarly, rapid fluctuations in $u$ can now be discerned during most of the time series [Fig. 2(c)]. Finally, at At $=1.19 \times 10^{-2}, u$ fluctuated rapidly with no apparent periodicity and $\left\langle w^{2}\right\rangle_{x}^{1 / 2}$ remained high at all times, characteristic of uniform turbulence [Fig. 2(d)].

The above discussion segregates flow regimes by At, which quantifies the initial density contrast. Under sufficiently turbulent conditions, however, the local density contrast at the fronts decays over time, causing the fronts to decelerate $[22,26]$. It seems likely that such flows evolve from turbulent to intermittent, provided that the tube is sufficiently long. Such a transition was captured at At $=4.0 \times 10^{-3}$. As described above, early times were dominated by elevated $\left\langle w^{2}\right\rangle_{x}^{1 / 2}$ and rapid fluctuations of $u$. At $t \gtrsim 150 \mathrm{~s}$, however, extended periods of suppressed $\left\langle w^{2}\right\rangle_{x}^{1 / 2}$ emerged [Fig. 2(g)].

The transition from laminar flow to uniform turbulence is often parameterized by the turbulent fraction $\gamma$, defined as the fraction of time during which the flow is turbulent. During each relaminarization phase, the rms of $w$ at the tube axis, $\left\langle w^{2}\right\rangle_{x}^{1 / 2}(z=0, t)$, taken as the mean $\left\langle w^{2}\right\rangle_{x}^{1 / 2}$ over $|\widetilde{z}|<0.05$, decayed to $\simeq 0.1 \mathrm{~cm} \mathrm{~s}^{-1}$. This limit serves as a convenient threshold against which flow at a given time may be classified as turbulent or laminar. $\gamma$ may then be calculated as the temporal average of the function

$$
I(t)=\left\{\begin{array}{ll}
1 & \text { if }\left\langle w^{2}\right\rangle_{x}^{1 / 2}(0, t)>w_{c} \\
0 & \text { otherwise }
\end{array},\right.
$$

where $w_{c}$ is the threshold.

Figure 3 presents $\gamma$ for $w_{c}=0.12 \mathrm{~cm} \mathrm{~s}^{-1}$ as a function of At. $\gamma$ increases monotonically with At and reaches its upper limit, $\gamma=1$, at At $\gtrsim 4 \times 10^{-3}$. Note that a $\pm 10 \%$ change in $w_{c}$, depicted by the vertical bars, does not alter the qualitative dependence of $\gamma$ on At. The sharp increase in $\gamma$ suggests that the intermittent regime investigated in this paper occurs, at least for $\theta=45^{\circ}$ considered presently, only within a relatively narrow range of At.

\section{B. Laminar-turbulent cycles}

We now consider the temporal evolution of the streamwise velocity, the turbulence intensity, and the strength of the stratification during laminar-turbulent cycles. For convenience, the discussion is in terms of variables that only vary with time. Specifically, the streamwise flow is characterized by

$$
\Delta U(t)=\langle u\rangle_{x}\left(\widetilde{z}^{+}(t), t\right)-\langle u\rangle_{x}\left(\widetilde{z}^{-}(t), t\right),
$$

where $\widetilde{z}^{-}(t)$ and $\widetilde{z}^{+}(t)$ are the transverse coordinates corresponding to minimum and maximum $\langle u\rangle_{x}$, respectively. Turbulence is characterized by $\left\langle w^{2}\right\rangle_{x}^{1 / 2}(0, t)$ and the stratification by the transverse density gradient at $\widetilde{z}=0$. Such gradients are denoted by $\partial /\left.\partial z\right|_{0}$ and are estimated as the gradient of the line of best fit over $\widetilde{z}^{-} / 2<\widetilde{z}<\widetilde{z}^{+} / 2$. Time is nondimensionalized by the period corresponding to the global 

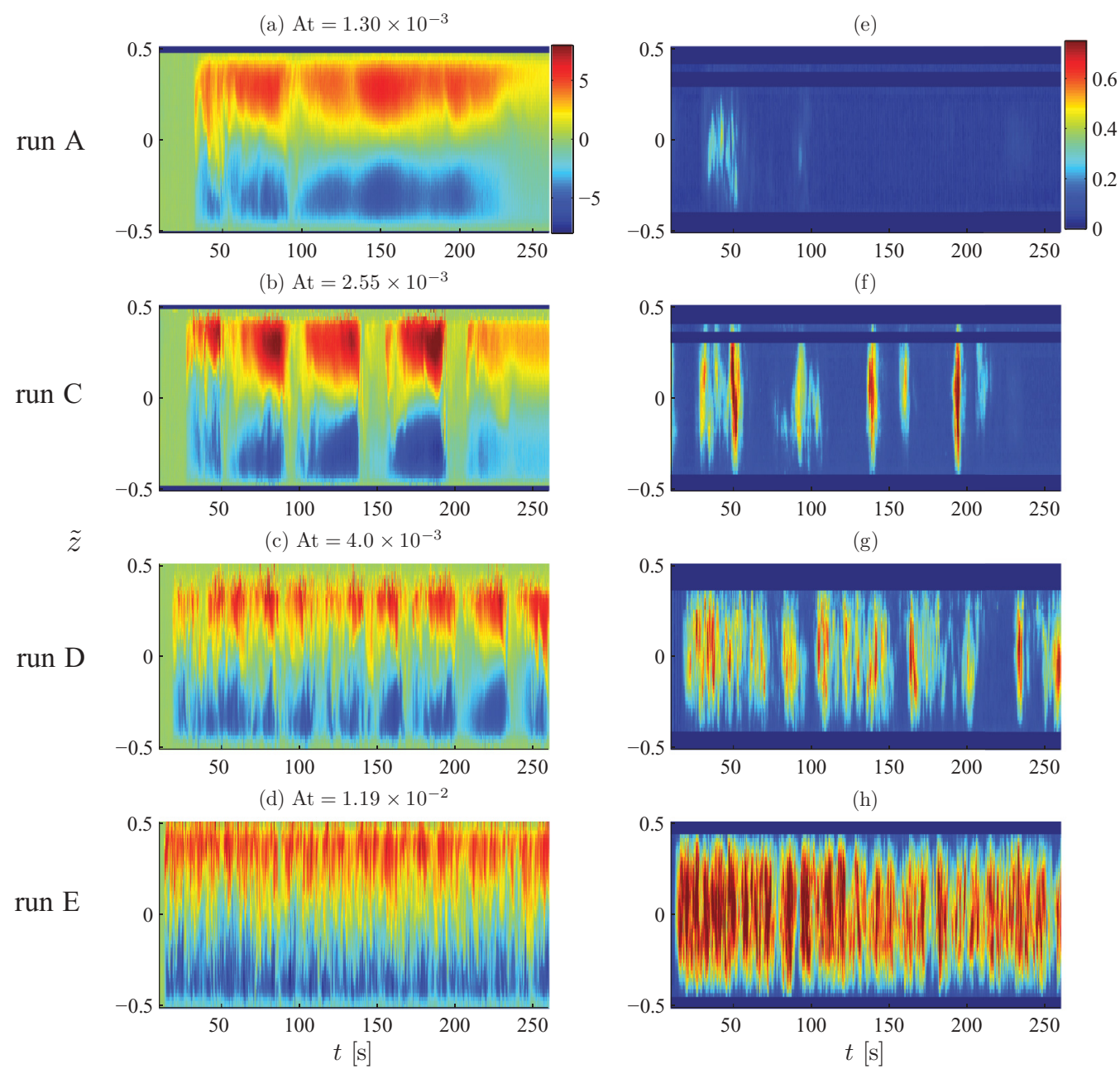

FIG. 2. (Color) The temporal evolution of $u\left[\mathrm{~cm} \mathrm{~s}^{-1}\right]$ at selected $x$ (a)-(d) and $\left\langle w^{2}\right\rangle_{x}^{1 / 2}\left[\mathrm{~cm} \mathrm{~s}^{-1}\right]$ (e)-(h). From top to bottom, the figures show runs $[(\mathrm{a}),(\mathrm{e})] \mathrm{A},[(\mathrm{b}),(\mathrm{f})] \mathrm{C},[(\mathrm{c}),(\mathrm{g})] \mathrm{D}$, and [(d), (h)] E in order of increasing At. The horizontal axes each extend from $t=10$ to $260 \mathrm{~s}$. The same color map is used for (a)-(d) and (e)-(h), which is displayed to the right of (a) and (e).

Brunt-Väisälä frequency [5],

$$
\tau_{N}=\frac{2 \pi}{\sqrt{2 g \mathrm{At} \sin \theta / d}},
$$

and denoted by $\widetilde{t}\left(=t / \tau_{N}\right)$.

For conciseness, the following discussion will be restricted to run $\mathrm{C}$, which exhibits multiple, distinct laminar-turbulent cycles and is thus convenient for the identification of salient features. The evolution of the parameters within the one laminar-turbulent cycle observed in runs B and D is qualitatively the same.

\section{Ramp-cliff pattern of mean streamwise velocity}

Figure 4(a) illustrates the characteristic ramp-cliff pattern of the temporal evolution of the axial flow during each relaminarization-turbulent cycle. Following the arrival of the front in the imaging region at $\widetilde{t}=5.4$, three rampcliff cycles can be readily identified, during which $\Delta U(t)$ [Eq. (3)] increases gradually from its local minimum of 2 to $3 \mathrm{~cm} \mathrm{~s}^{-1}$ to $\Delta U=14$ to $16 \mathrm{~cm} \mathrm{~s}^{-1}$, then decreases rapidly to the subsequent local minimum. Interestingly, the local minima correspond to $\Delta U \approx 2 V_{f}$, suggesting that the front velocity is governed primarily by the low $\Delta U$ periods. The increase in $\Delta U$ initiated at $\widetilde{t}=43.4$ does not develop into a ramp. This is interpreted as an arrest of the ramp development due to interference from the return flow initiated when the propagating fronts reach the ends of the tube. This is consistent with the reduction of $u$ at the end of the run $[t>210 \mathrm{~s}$, Fig. 2(b)] and implies that the number of laminar-turbulent cycles was limited by the length of the tube.

Each "cliff" phase begins with the onset of the breakdown of billows, at $\widetilde{t}=\left(\widetilde{t}_{c}^{1}, \widetilde{t}_{c}^{2}, \widetilde{t}_{c}^{3}\right)=(18,29,40.4)$, which is depicted by a sharp rise in $\left\langle w^{2}\right\rangle_{x}^{1 / 2}(0, t)$ to its local maximum [Fig. 4(b), dashed line]. As the billows continue to break down into finer structures, $\left\langle w^{2}\right\rangle_{x}^{1 / 2}(0, t)$ begins to decrease. The cliff phase ends, i.e., $\Delta U$ drops to its local minimum (dotted line), while $\left\langle w^{2}\right\rangle_{x}^{1 / 2}(0, t)$ is still above levels observed in laminar states. The elevated $\left\langle w^{2}\right\rangle_{x}^{1 / 2}(0, t)$ is consistent with the visual observation of turbulent mixing and reflects the conversion of mean kinetic energy into turbulent kinetic energy. $\left\langle w^{2}\right\rangle_{x}^{1 / 2}(0, t)$ broadly decays to $\simeq 0.1 \mathrm{~cm} \mathrm{~s}^{-1}$ during the "ramp" phase, consistent with observations of flow relaminarization. Further, 


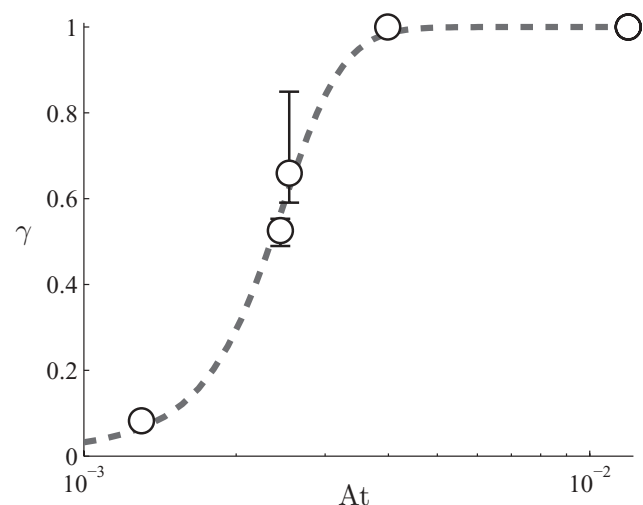

FIG. 3. Turbulent fraction $\gamma$ [Eq. (2), $w_{c}=0.12 \mathrm{~cm} \mathrm{~s}^{-1}$ ] as a function of At. The temporal average of $I(t)$ was taken over $50<$ $t<200 \mathrm{~s}$. The dashed line is intended only as a guide to the eye. The upper and lower limits of the vertical bars correspond to $w_{c}=1.08$ and $1.32 \mathrm{~cm} \mathrm{~s}^{-1}$, respectively. Where bars are not visible, the variation is smaller than the marker. The two data points at At $=1.19 \times 10^{-2}$ coincide exactly and cannot be distinguished in the figure.

we determined from a frame-by-frame inspection of the velocity field that, in each cycle, the onset of shear-induced instability leading to turbulence (dash-dotted line) occurs at $\tilde{t}=\left(\widetilde{t}_{b}^{1}, \widetilde{t}_{b}^{2}, \widetilde{t}_{b}^{3}\right)=(16,28,39)$, i.e., before $\Delta U$ reaches its local maximum. These observations indicate that flow acceleration occurs concurrently with relaminarization, and that the streamwise flow is suppressed by the breakdown of billows and subsequent turbulent motion at the scale of the tube diameter.

In cycles 2 and 3, the cliff phase is not followed immediately by a ramp phase of the subsequent cycle, but by a period of decaying turbulence. During this period, transverse velocity fluctuations, $\left\langle w^{2}\right\rangle_{x}^{1 / 2}(0, t)$, and the density gradient, $-\partial\langle\widetilde{\rho}\rangle_{x} /\left.\partial \widetilde{z}\right|_{0}$, decay to their respective minima, while $\Delta U$ remains at its minimum value. We believe that this phase did not take place at the end of cycle 1 because the ramp phase in the second cycle was initiated exactly at the end of the cliff phase, i.e., $\tilde{t}_{a}^{2}=\tilde{t}_{d}^{1}$.

This period of decaying residual turbulence ends with the arrival of pockets of fluid of concentration near that of the original, unmixed fluid. These pockets take on, at the local scale, the canonical profile of a gravity current head, with billows attached to its wake (see, e.g., Ref. [27]). The arrival of these current heads was identified visually at $\tilde{t}=\tilde{t}_{a}^{2}=20.2$, $\tilde{t}_{a}^{3}=32.4$, and $\tilde{t}_{a}^{4}=43.4$ [Fig. 4(a)] and is associated with a sudden, sharp increase in $\left\langle w^{2}\right\rangle_{x}^{1 / 2}(0, t)$ and $\Delta U$, caused by the displacement of well-mixed, relatively slow fluid upstream of the front. $\left\langle w^{2}\right\rangle_{x}^{1 / 2}(0, t)$ is maintained above laminar-phase levels for some time by the billows trailing behind the head, then decays once again. The simultaneous, abrupt increase in $-\partial\langle\widetilde{\rho}\rangle_{x} /\left.\partial \widetilde{z}\right|_{0}$ reflects the arrival of relatively unmixed fluid into fluid that is well mixed having just undergone the turbulent phase. This reestablishment of the stratification during each relaminarization phase [Fig. 4(d)], despite its having been eroded during the preceding turbulent phase, gives rise to the most striking feature of Fig. 4: the apparent absence of a global decay in the maximum shear and stratification over successive ramps.
The evolution of $\Delta U$ discussed above is reminiscent of the ramp-cliff spatial variation of the temperature in unbounded stably stratified shear flows $[3,4]$ and of the peak streamwise velocity in uniform pipe flows, which alternate between laminar and turbulent states at Reynolds numbers between 2300 and 2600 [28]. The resemblance is interesting, given that the associated physical phenomena in the latter are fundamentally different. In unbounded flows studied in Refs. $[3,4]$, a ramp is a signature of a $\mathrm{KH}$ billow at an early stage of overturning and a cliff represents the region between adjacent billows. In contrast, in pipe flows and in flows considered presently, the ramps and cliffs occur largely in the absence of KH billows.

\section{Transverse profiles of mean velocity and density}

Figure 5 compares the transverse profiles of streamwise velocity and density in laminar and turbulent states in run $\mathrm{C}$ to those of permanently laminar (run A) and uniformly turbulent flows (run E), respectively. To facilitate the comparison between the differences in flow speed and absolute densities observed at different At, we focus on the normalized streamwise velocity, $\langle u\rangle_{x}(z, t) / \Delta U(t)$, and the density relative to its value at $z=0$ and normalized by the original density contrast,

$$
\langle\widetilde{\rho}\rangle_{x}(z, t)-\langle\widetilde{\rho}\rangle_{x}(0, t)
$$

where

$$
\langle\widetilde{\rho}\rangle_{x}(z, t)=\frac{\langle\rho\rangle_{x}(z, t)-\left(\rho_{1}+\rho_{2}\right) / 2}{\rho_{2}-\rho_{1}} .
$$

$\langle\widetilde{\rho}\rangle_{x}(0, t)$ in Eq. (5) is estimated as the average of $\langle\widetilde{\rho}\rangle_{x}$ over $|\widetilde{z}|<0.05$. Specifically, Fig. 5 presents these profiles at the end of two flow relaminarization phases $(\widetilde{t}=28.1,39.2)$ and during the subsequent turbulent decay phase $(\widetilde{t}=30.9,43.0)$ in run $\mathrm{C}$. For runs $\mathrm{A}$ and $\mathrm{E}$ (thick dashed lines), we take advantage of the stationarity of the flow and consider instead $\langle u\rangle_{x, t}(z)$, the long-time temporal-average of $\langle u\rangle_{x}$, normalized by its peak-to-peak difference. Note that we do not temporally average the density.

We first consider the velocity profiles. All $\langle u\rangle_{x}$ exhibit the canonical profile of an exchange flow in closed systems: it is approximately antisymmetric about $\widetilde{z}=0$, with a maximum at $\widetilde{z}=\widetilde{z}^{+}(>0)$ and a minimum at $\widetilde{z}=\widetilde{z}^{-}(<0)$. The maximum and the minimum $\langle u\rangle_{x}$ occur at about the same distance from the tube axis, i.e., $\widetilde{z}^{+} \approx-\widetilde{z}^{-}$.

The excellent coincidence between the instantaneous velocity profiles at the selected times in run $\mathrm{C}$ (thin lines) and the time-averaged profiles of runs $\mathrm{A}$ and $\mathrm{E}$ (thick dashed lines) is readily apparent in Fig. 5(a) and 5(b). $\langle u\rangle_{x}$ at $\widetilde{t}=28.1$ and 39.2 and $\langle u\rangle_{x, t}$ in run $\mathrm{A}$ all exhibit a gradual variation of its gradient with $z$ and extrema at $\widetilde{z}^{*} \equiv\left(\widetilde{z}^{+}-\widetilde{z}^{-}\right) / 2 \approx 0.30$, consistent with laminar flows at larger $\theta$ reported in Ref. [21]. Similarly, $\langle u\rangle_{x}$ at $\tilde{t}=30.9$ and 43.0 and $\langle u\rangle_{x, t}$ in run E exhibit a linear dependence on $\widetilde{z}$ in the region $|\widetilde{z}| \leqslant 0.3$ with the extrema at $\widetilde{z}^{*}=0.37-0.38$, consistent with uniformly turbulent flows at other $(\theta$, At) reported elsewhere (e.g., Ref. [21]). Between these two states, $\widetilde{z}^{*}$ evolves in a ramp-cliff pattern similar to that of $\Delta U$ [Fig. 4(c)]. 


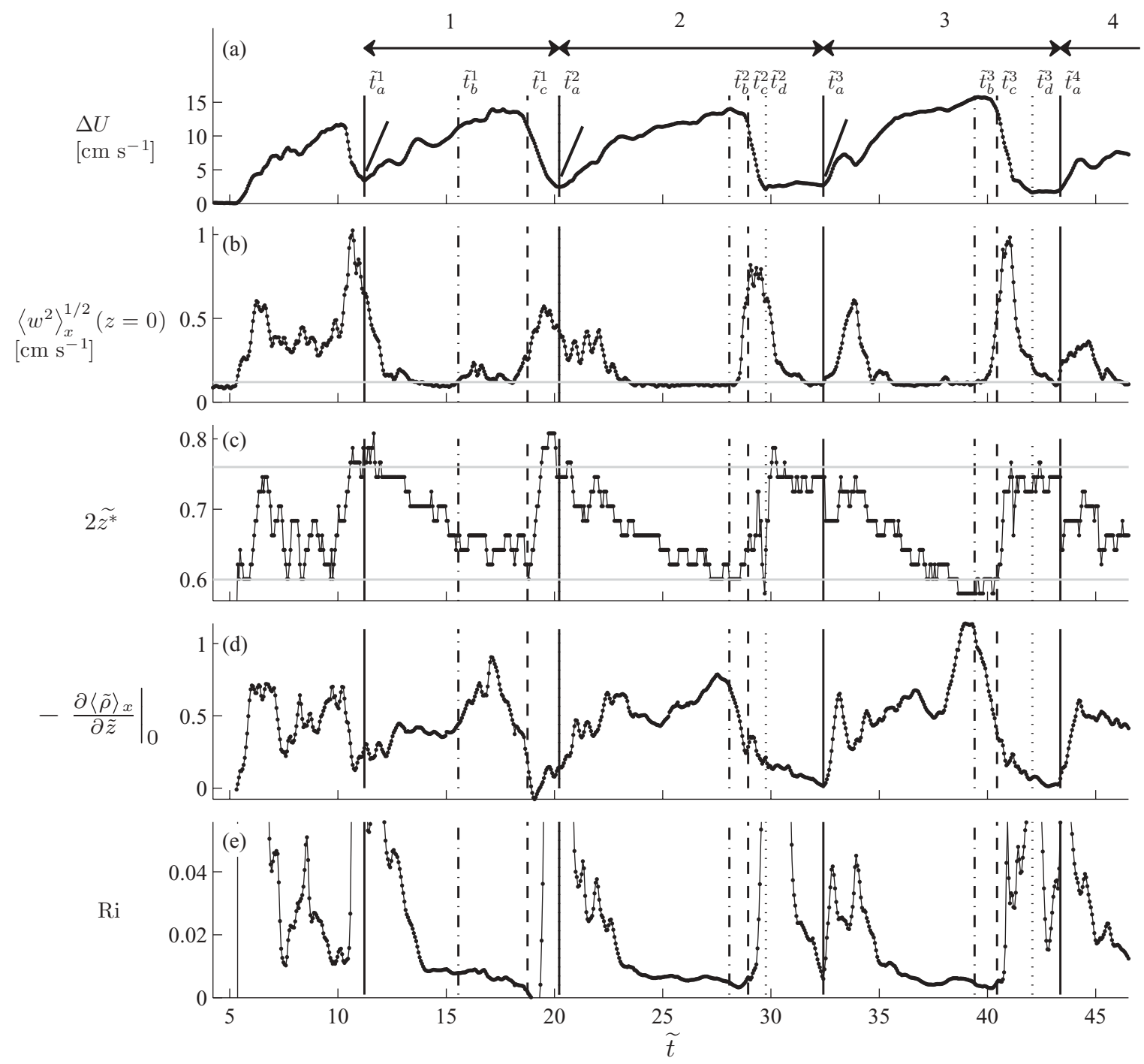

FIG. 4. Temporal evolution of (a) $\Delta U$, (b) $\left\langle w^{2}\right\rangle_{x}^{1 / 2}(0, t)$, (c) the transverse separation between the extrema of $\langle u\rangle_{x}$, (d) - $\partial\langle\widetilde{\rho}\rangle_{x} /\left.\partial \widetilde{z}\right|_{0}$, and (e) the instantaneous Richardson number, Ri [Eq. (14)], in run C. The three ramp-cliff cycles (and the beginning of the fourth) are numbered at the top. $\widetilde{t}_{a}^{i}(i=1,2,3, \ldots)$ corresponds to the arrival of relatively unmixed fluid that initiates the ramp phase (solid line); $\widetilde{t}_{b}^{i}, \widetilde{t}_{c}^{i}$, and $\widetilde{t}_{d}^{i}$ correspond to the onset of instability (dash-dotted), the onset of the breakdown of billows (dashed), and the local minima in $\Delta U$ (dotted). $\widetilde{t}_{d}^{1}$ coincides with $\widetilde{t}_{a}^{2}$. The oblique, solid line after $\widetilde{t}_{a}^{i}$ in (a) depicts the theoretical acceleration of a "free-fall" [Sec. III B3, Eq. (9)]. The horizontal line in (b) depicts the threshold $w_{c}=0.12 \mathrm{~cm} \mathrm{~s}^{-1}$ [Eq. (2)]. Horizontal lines in (c) depict $\widetilde{z}^{*}$ in laminar flows $\left(2 \widetilde{z}^{*}=0.60\right)$ and in uniformly turbulent flows $\left(2 \widetilde{z}^{*}=0.76\right)$.

Like those of $\langle u\rangle_{x} / \Delta U$, the transverse profile of the density contrast, $\langle\widetilde{\rho}\rangle_{x}-\langle\widetilde{\rho}\rangle_{x}(0, t)$, in run $\mathrm{C}$ at the end of relaminarization phases and during the turbulence decay phase resemble that in permanently laminar and uniformly turbulent flows, respectively. The difference in $\langle\widetilde{\rho}\rangle_{x}-\langle\widetilde{\rho}\rangle_{x}(0, t)$ between the upper and lower layers was large $(\simeq 0.8)$ in laminar states [Fig. 5(c)] but was subsequently eroded to near-zero during the turbulent decay phase [Fig. 5(d)]. The coincidence of the profiles in Fig. 5(c) illustrates the reestablishment of the stratification during each ramp phase [cf. Fig. 4(d)].

\section{Characteristic scales of the ramp-cliff cycle}

To validate our physical understanding of the dominant processes that control the laminar-turbulent alternation, we consider here the characteristic scale of the four phases of a ramp-cliff cycle (Sec. III B1):

(1) Flow relaminarization and acceleration (the ramp)

(2) The growth of the KH instability

(3) Flow deceleration (the cliff) and

(4) The turbulent decay phase.

Specifically, we define an acceleration scale for the ramp phase and the time scale for the duration of the KH billow growth, the duration of the cliff phase, and the arrival of the current of unmixed fluid that initiates the next cycle.

We first consider the ramp phase. For simplicity, we assume that viscosity is negligible, that the density is uniform in each layer, and that the flow is two-layered, laminar, and parallel. With these assumptions, the upper (lower) current may be approximated as a "free rise" ("free fall") governed by the 

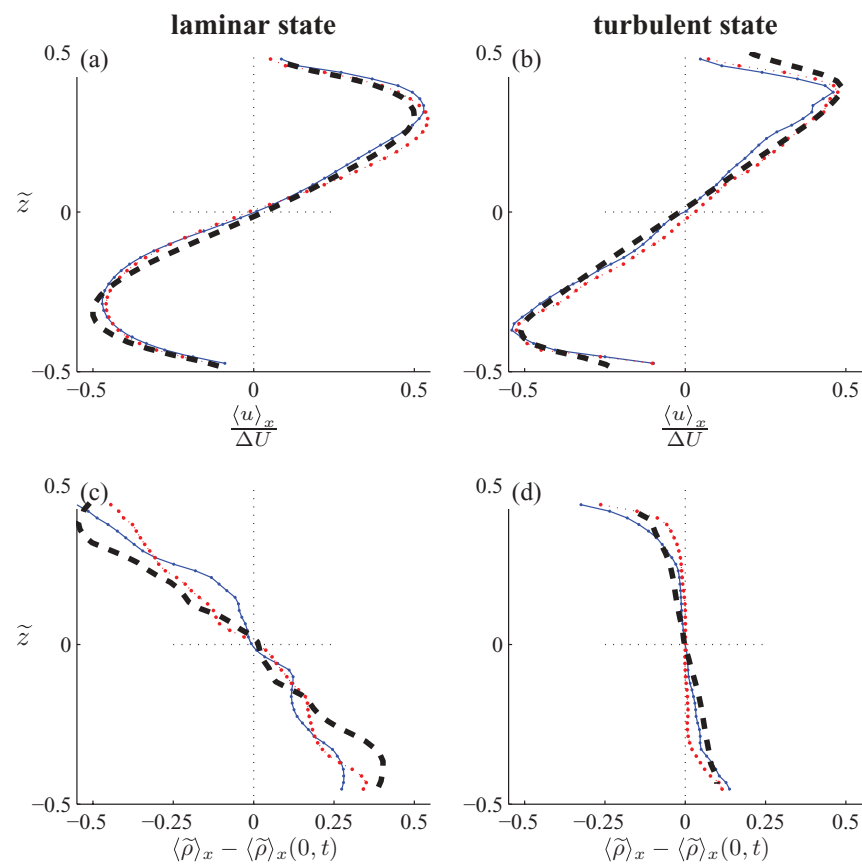

FIG. 5. (Color online) Transverse profiles of $\langle u\rangle_{x}(z, t) / \Delta U(t)$ and $\langle\widetilde{\rho}\rangle_{x}(z, t)-\langle\widetilde{\rho}\rangle_{x}(0, t)$ at the end of a relaminarization phase [(a), (c)] and during the subsequent turbulence decay phase $[(\mathrm{b}),(\mathrm{d})]$ in run $\mathrm{C}$. $\tilde{t}=28.1$ [solid blue line (a), (c)], 30.9 [solid blue line (b), (d)], 39.2 [dotted red line (a), (c)], 43.0 [dotted red line (b), (d)]. Thick dashed lines represent the corresponding profiles in permanently laminar [run A (a), (c)] and uniformly turbulent flows [run E (b), (d)]; $\langle u\rangle_{x}$ was temporally averaged over $120 \leqslant t \leqslant 199 \mathrm{~s}$ (run A) and $25 \leqslant t<107$ $\mathrm{s}(\operatorname{run} \mathrm{E}) ; t=199 \mathrm{~s}(\mathrm{c})$ and $t=106 \mathrm{~s}(\mathrm{~d})$.

balance between the inertial acceleration and the local density contrast:

$$
\begin{aligned}
\frac{\mathrm{d}}{\mathrm{d} t}\left[\langle u\rangle_{x}\left(\widetilde{z}^{+}(t), t\right)\right] & =-\frac{\mathrm{d}}{\mathrm{d} t}\left[\langle u\rangle_{x}\left(\widetilde{z}^{-}(t), t\right)\right] \\
& \simeq g \cos \theta\left(\frac{\langle\rho\rangle_{x}^{-}-\langle\rho\rangle_{x}^{+}}{\langle\rho\rangle_{x}^{-}+\langle\rho\rangle_{x}^{+}}\right),
\end{aligned}
$$

where $\langle\rho\rangle_{x}^{-}(t)$ and $\langle\rho\rangle_{x}^{+}(t)$ are the density of the denser (hence $z<0)$ current and lighter $(z>0)$ current, respectively. To calculate the theoretical acceleration during a ramp phase, we approximate $\langle\rho\rangle_{x}^{-}(t)$ and $\langle\rho\rangle^{+}(t)$ by $\langle\rho\rangle_{x}\left(\widetilde{z}^{-}\right)$and $\langle\rho\rangle_{x}\left(\widetilde{z}^{+}\right)$, respectively, and take a temporal average over the ramp phase. Then, Eqs. (7) and (8) reduce to

$$
\frac{\mathrm{d} \Delta U}{\mathrm{~d} t} \simeq 2 g \cos \theta \overline{\left[\frac{\langle\rho\rangle_{x}\left(\widetilde{z}^{-}\right)-\langle\rho\rangle_{x}\left(\widetilde{z}^{+}\right)}{\langle\rho\rangle_{x}\left(\widetilde{z}^{-}\right)+\langle\rho\rangle_{x}\left(\widetilde{z}^{+}\right)}\right]},
$$

where the overbar denotes the temporal average from $\widetilde{t}_{a}^{i}$ to $\widetilde{t}_{b}^{i}$ for the $i$ th cycle. Applying the density measurements to Eq. (9) yields $\mathrm{d} \Delta U / \mathrm{d} t \simeq 1.6 \pm 0.1 \mathrm{~cm} \mathrm{~s}^{-2}$ ( \pm standard deviation $)$ for cycles 1-3 [oblique solid lines, Fig. 4(a)]. The prediction agrees to within an order of magnitude with the acceleration during the early stages of the flow relaminarization phase; the consistent overestimation is likely to be due to viscous effects.

The growth rate of $\mathrm{KH}$ billows is typically governed by the shear that gives rise to the instability, whose characteristic time may be calculated directly from the measurements of the local shear at the onset of instability as

$$
\tau_{s}=\left(\left.\frac{\partial\langle u\rangle_{x}}{\partial z}\right|_{0}\right)^{-1} .
$$

In run $\mathrm{C}, \partial\langle u\rangle_{x} /\left.\partial z\right|_{0}=10.0,16.0$, and $20.5 \mathrm{~s}^{-1}$ at $\tilde{t}_{b}^{1}, \tilde{t}_{b}^{2}$, and $\tilde{t}_{b}^{3}$, respectively. These values correspond to $\tau_{s}=(0.01$ to 0.02$) \tau_{N}$. In contrast, the observed growth period of the billows, taken as the period between the onset of instability (dash-dotted lines, Fig. 4) and the subsequent breakdown of $\mathrm{KH}$ billows (dashed lines), ranged between (0.9 to 3.2$) \tau_{N}$ in the three cycles. This extension of the billow growth period by a factor of 60 to 150 beyond the time scale of the shear is attributed to a stabilizing effect of the confinement and of the viscosity.

The duration of the cliff is expected to be governed by the turbulent mixing that follows the breakdown of the $\mathrm{KH}$ billows. The time scale of the latter may be calculated as

$$
\tau_{m}=\frac{d}{\left(\overline{\left\langle w^{2}\right\rangle_{x}}\right)^{1 / 2}(z=0)},
$$

where the overbar denotes a temporal average during each cliff, starting at by the onset of the breakdown of KH billows (dashed lines) and ending at the subsequent local minimum in $\Delta U$ (dotted lines). For the turbulent bursts in cycles 2 and $3, \tau_{m}=(0.58,0.67) \tau_{N}$. These values are in good agreement with the observed duration, $\left(\tilde{t}_{d}^{2}-\tilde{t}_{c}^{2}, \tilde{t}_{d}^{3}-\widetilde{t}_{c}^{3}\right)=(0.8,1.6) \tau_{N}$. Similarly, the cliffs lasted for $1.1 \tau_{m}$ and $2.0 \tau_{m}$ in runs B and $\mathrm{D}$, respectively (not shown). These results indicate that $\tau_{m}$ is an adequate characterization of the duration of the cliff.

Finally, we consider the duration of the turbulent decay phase that follows the cliff. Recall that this decay phase is terminated by the passage of a local gravity current of relatively unmixed fluid through the imaging region (Sec. III B1). We believe that these transient gravity currents originate in the undisturbed regions beyond the macroscopic flow fronts, then propagate upstream during a temporary weakening of turbulence in their vicinity (see Ref. [22]). The path length of this transient current head from its origin to the imaging region may be approximated as $x \simeq V_{f} t$, where the speed of the macroscopic flow fronts, $V_{f}$, is approximated by its asymptotic (long-time) value. This value is interpolated from previous [20] measurements to be $1.24 \mathrm{~cm} \mathrm{~s}^{-1}$ for run C (Table I). The time required for the gravity current to travel this distance is

$$
\widetilde{\tau}_{g}(t)=\frac{\tau_{g}(t)}{\tau_{N}}=\frac{V_{f}}{u_{g}} \widetilde{t},
$$

where $u_{g}$ is the propagation speed of a gravity current head. $u_{g}$ may be approximated by the local maximum in $u(x, z, t)$ at $x$ corresponding to the middle of the measurement region [cf. Fig. 2(b)], which yields $u_{g} / V_{t}=2.7$ and 2.5 for the gravity current that arrives at $\widetilde{t}_{a}^{3}$ and $\tilde{t}_{a}^{4}$, respectively. Applying these values to Eq. (12) yields $\widetilde{\tau}_{g}\left(\widetilde{t}_{a}^{3}\right)=8.0$ and $\tilde{\tau}_{g}\left(\tilde{t}_{a}^{4}\right)=11.6$. These values overestimate the duration of the turbulence decay phases in cycles 2 and 3 by a factor of 3 and 9 , respectively $\left(\widetilde{t}_{a}^{3}-\widetilde{t}_{d}^{2}=2.7 ; \tilde{t}_{a}^{4}-\widetilde{t}_{d}^{3}=1.3\right)$. This overestimation is attributed largely to an underestimation of $u_{g}$ : contrary to the assumption of fixed speed, the pure fluid 
current is expected to have decelerated over time due to viscosity.

\section{Richardson and Reynolds numbers at the onset of instability}

Finally, we examine the extent to which the onset of instability in laminar-turbulent cycles may be predicted from the local Reynolds and gradient Richardson numbers. The instantaneous Reynolds number is calculated as

$$
\operatorname{Re}(t)=\Delta U(t) \frac{d}{v} .
$$

The instantaneous gradient Richardson number is defined here as

$$
\operatorname{Ri}(t)=\left.\frac{-g \sin \theta}{\left.\langle\rho\rangle_{x}\right|_{0}} \frac{\partial\langle\rho\rangle_{x}}{\partial z}\right|_{0}\left(\left.\frac{\partial\langle u\rangle_{x}}{\partial z}\right|_{0}\right)^{-2},
$$

where $\left.\langle\rho\rangle_{x}\right|_{0}(t)$ is taken as the mean $\langle\rho\rangle_{x}$ in the region $\widetilde{z}^{-} / 2<$ $\tilde{z}<\widetilde{z}^{+} / 2$.

While Eq. (14) does not preclude the computation of Ri under nonlaminar conditions, gradients of spatially averaged parameters do not capture the rapidly fluctuating velocity and density fields characteristic of turbulent states. $\partial\langle\rho\rangle_{x} /\left.\partial z\right|_{0}$ and $\mathrm{Ri}$ in turbulent states are displayed in Fig. 4(d) and 4(e) for the purpose of qualitative comparison only.

\section{Richardson and Reynolds numbers}

During flow relaminarization, both shear and stratification strengthen [Fig. 4(a) and 4(d)]. Combined, the growth of shear dominates, such that $\mathrm{Ri}$ first decreases rapidly to $\approx 0.01$, then decays very slowly so that is is nearly constant at values ranging from $5 \times 10^{-3}$ to $8 \times 10^{-3}$ at the onset of instability [Fig. 4(e)]. In this section we examine more closely the variations of $\mathrm{Ri}$ and $\mathrm{Re}$ during relaminarization and up to the onset of instability.

Figure 6 displays the temporal evolution of $\operatorname{Re}(t)$ and $\operatorname{Ri}(t)$ during each relaminarization phase in runs B, C, and D (thin solid lines). Each relaminarization phase ends with the onset of instability (solid markers). Clearly, Re increases and Ri decreases during relaminarization. Moreover, the Ri-Re curves at different At are indistinguishable, possibly due to their narrow range. In the discussion that follows, the data from the five relaminarization phases depicted in Fig. 6 are treated collectively.

$\mathrm{Re}$ and $\mathrm{Ri}$ at the onset of instability, denoted by $\mathrm{Re}_{i}$ and $\mathrm{Ri}_{i}$, vary from 2200 to 3100 and from $4.5 \times 10^{-3}$ to $7.8 \times 10^{-3}$, respectively. These $\mathrm{Ri}_{i}$ are one order of magnitude smaller than the near-constant threshold of about 0.20 predicted by the neutral stability curve of Defina et al. [17] for $\operatorname{Re}_{i}>2000$. A similar disagreement with the theory has been reported in stratified shear flows generated in a Thorpe tilting tank at At ranging from $2.5 \times 10^{-3}$ to $17.5 \times 10^{-3}$, of which two are depicted in Fig. $6(\circ, \diamond)$ [17]. Furthermore, Ref. [17] reports $\mathrm{Re}_{i}$ ranging from 1290 to as high as 5340 (not shown). In this context, we interpret the variation in $\operatorname{Re}_{i}$ in the present experiments not as a manifestation of experimental uncertainty, but as a salient property of strongly confined stratified shear flows that are considered in Ref. [17] and the present study.

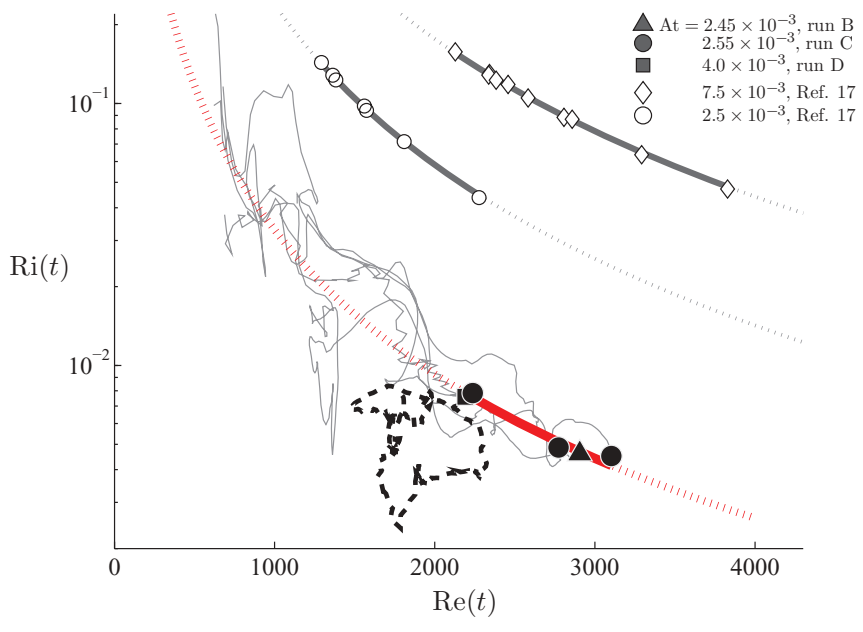

FIG. 6. (Color online) (Re, Ri) at the onset of instability in runs B $(\boldsymbol{\Delta}), \mathrm{C}(\bullet)$, and D ( $\boldsymbol{\square})$. The thick red line is the least-squares best-fit power function to all five points. Thin solid lines depict the evolution of Re and Ri during each relaminarization phase. The thick dashed line depicts run A $(t=120-199 \mathrm{~s})$, in which the flow remained laminar. Open markers depict $\left(\mathrm{Re}_{i}, \mathrm{Ri}_{i}\right)$ in inclined rectangular tubes at $\mathrm{At}=2.5 \times 10^{-3}(\circ)$ and $7.5 \times 10^{-3}(\diamond)$ reported by Defina et al . (Ref. [17], $\theta=80^{\circ}$ to $88.4^{\circ}$ ), where $\mathrm{Ri}_{i}$ was calculated taking $\sin \theta=$ 0.99 and $\operatorname{Re}_{c}$ was estimated as $2 \mathrm{Re}_{i}^{\mathrm{DLS}}$, where $\mathrm{Re}_{i}^{\mathrm{DLS}}$ is the Reynolds number as defined in Ref. [17]. Thick gray lines are the corresponding least-squares best-fit power functions.

By definition, Ri varies as $\left(\partial\langle u\rangle_{x} /\left.\partial z\right|_{0}\right)^{-2}$ [Eq. (14)]. If the decay in $\mathrm{Ri}_{i}$ simply reflects the concurrent increase in Reynolds number, with negligible contribution from variations in stratification, the $\mathrm{Re}$ dependence of $\mathrm{Ri}_{i}$ will be $\mathrm{Ri}_{i} \propto \mathrm{Re}_{i}^{-2}$ approximately. While the present data span a narrow range of $\mathrm{Re}_{i}$, they are consistent with such a power law; a leastsquares fit is of the form $\mathrm{Ri}_{i} \propto \mathrm{Re}_{i}^{-1.7 \pm 0.2}$ (thick red line). Similarly, the best-fit exponent to the data of Defina et al. [17] is $-2.04 \pm 0.02$ (standard error).

Interestingly, despite their resemblance, the instability that terminated the relaminarization shown for run $\mathrm{B}(\boldsymbol{\Delta})$ did not develop into a turbulent burst unlike the other four relaminarization phases shown in Fig. 6, suggesting that the onset of a turbulent burst is controlled by properties other than $\mathrm{Ri}$ and $\mathrm{Re}$.

\section{Criterion for the onset of instability}

Figure 6 also compares $\mathrm{Ri}_{i}$ in runs $\mathrm{B}-\mathrm{D}$ with $\mathrm{Ri}$ in run $\mathrm{A}$, in which the flow exhibited long waves but did not develop into $\mathrm{KH}$ billows (thick dashed line). Ri oscillated between $2.4 \times 10^{-3}$ and $8.4 \times 10^{-3}$, indicating that the flow remained laminar at Richardson numbers below $\mathrm{Ri}_{i}$ at which instability first appeared at larger At. Thus a single Ri does not distinguish flows that remain laminar from those which subsequently exhibit elevated $\left\langle w^{2}\right\rangle_{x}^{1 / 2}(0, t)$. Combined with results presented in Secs. III B1 and III B2, this suggests that stratification does not directly control the onset of instability.

Instead, Fig. 6 and the ramp-cliff evolution of $\Delta U$ shown in Fig. 4(a) suggest that the instability is controlled primarily by the instantaneous Re. The Reynolds number immediately prior to the onset of instability ranges from $\mathrm{Re}_{i}=2200$ to 
3100 in runs B-D, while Re oscillates between 1500 and 2300 in run A. Like the Ri-Re trajectories (Sec. III C1), $\operatorname{Re}_{i}$ does not exhibit a dependence on At. Therefore, despite some overlap, the two classes are largely segregated in Re, and an approximate threshold can be defined at $\mathrm{Re}=2200-2300$. The delay of instability to $\operatorname{Re}_{i}$ as high as 3100 in three of the five relaminarization (ramp) phases indicates that this threshold is a necessary, but insufficient, condition for the onset of instability under the experimental conditions considered presently.

As discussed in Sec. I, in many flow configurations there is a critical Reynolds number below which the flow is stable to all Richardson numbers and the stability is determined by the Reynolds number. In this framework, the threshold identified here may be interpreted as this critical Reynolds number at the limit of Ri approaching 0 . The one-order-of-magnitude difference between the critical $\mathrm{Re}$ in the present flow configuration and that of Defina et al. $(\mathrm{Re}=150)$ is attributed to the radial confinement in the present configuration. No-slip boundaries stabilize the flow [29], hence we expect the onset of instability in the present experiments to be delayed to higher Reynolds numbers (or lower Richardson numbers).

\section{CONCLUSIONS}

Lock-exchange flows in a circular tube inclined at $\theta=45^{\circ}$ exhibit two distinct states at At $=2.45 \times 10^{-3}$ to $4.0 \times 10^{-3}$ : parallel, laminar flow and turbulent flow characterized by intense transverse fluctuations. During each laminar-turbulent cycle, the flow accelerates as it relaminarizes and then decelerates rapidly with the breakdown of Kelvin-Helmholtz billows. Accordingly, each cycle is associated with a ramp-cliff variation in the peak streamwise velocity.

The coevolution of transverse stratification, shear, and transverse rms velocity suggests that, while the density contrast at the propagating fronts drives the exchange flow, transverse stratification does not directly control the onset of instability within the range of conditions considered in the present study. Instead, the onset of instability appears to be controlled by the Reynolds number. The present data suggest that a necessary criterion for the onset of instability is for $\mathrm{Re}$ to exceed 2200-2300, below which a ramp-cliff, laminar-turbulent cycle does not develop. Interestingly, this threshold is the same order of magnitude as the Reynolds number $(=2300)$ at which intermittent turbulence emerges in uniform pipe flows [28].

To the authors' knowledge, sustained alternation between turbulent and laminar states has not been documented previously in stratified shear flows. One interesting question that emerges from the present study is whether the onset of instability in each cycle is controlled locally, or if it is synchronized over the entire length of the exchange flow. Further insights require concurrent measurements at a scale comparable to the tube length.

\section{ACKNOWLEDGMENTS}

This material is based on work supported by the Agence Nationale pour la Recherche grant ANR-07-BLAN-0181. The authors thank Christian Borget and Rafael Pidoux for the construction of the experimental setup and the two anonymous reviewers for their comments.
[1] K. A. Browning, Quart. J. R. Met. Soc. 97, 283 (1971).

[2] W. Blumen, R. Banta, S. P. Burns, D. C. Fritts, R. Newsom, G. S. Poulos, and J. Sun, Dynam. Atmos. Oceans 34, 189 (2001).

[3] J. A. Whiteway, G. P. Klaassen, N. G. Bradshaw, and J. Hacker, Geophys. Res. Lett. 31, L02118 (2004).

[4] D. E. Wroblewski, O. R. Coté, J. M. Hacker, and R. J. Dobosy, J. Atmos. Sci. 64, 2521 (2007).

[5] J. S. Turner, Buoyancy Effects in Fluids (Cambridge University Press, Cambridge, UK, 1998).

[6] J. D. Woods, Radio Sci. 4, 1289 (1969).

[7] R. W. Garvine and J. D. Monk, J. Geophys. Res. 79, 2251 (1974).

[8] C. Sun, W. D. Smyth, and J. N. Moum, J. Geophys. Res. 103, 10323 (1998).

[9] W. R. Geyer and J. D. Smith, J. Phys. Oceanogr. 17, 1668 (1987).

[10] Y. Kitade, M. Matsuyama, and J. Yoshida, J. Oceanogr. 59, 845 (2003).

[11] E. Roget, J. Colomer, X. Casamitjana, and J. E. Llebot, Aquatic Sci. 55, 206 (1993).

[12] C. B. Lovstedt and L. Bengtsson, Water Resour. Res. 44, W10413 (2008).

[13] F. J. Rueda, S. G. Schladow, S. G. Monismith, and M. T. Stacey, J. Hydraul. Eng. 129, 82 (2003).

[14] F. K. Browand and C. D. Winant, Bound.-Lay. Meteorol. 5, 67 (1973).

[15] S. A. Thorpe, J. Geophys. Res. 92, 5231 (1987).
[16] K. S. Gage and W. H. Reid, J. Fluid Mech. 33, 21 (1968).

[17] A. Defina, S. Lanzoni, and F. M. Susin, Phys. Fluids 11, 344 (1999).

[18] J. O. Shin, S. B. Dalziel, and P. F. Linden, J. Fluid Mech. 521, 1 (2004).

[19] T. Séon, J. Znaien, D. Salin, J. P. Hulin, E. J. Hinch, and B. Perrin, Phys. Fluids 19, 123603 (2007).

[20] T. Séon, Ph.D. thesis, University of Paris 6, Orsay, France 2006.

[21] J. Znaien, F. Moisy, and J. P. Hulin, Phys. Fluids 23, 035105 (2011).

[22] Y. Hallez and J. Magnaudet, Phys. Fluids 20, 053306 (2008).

[23] T. Séon, J. Znaien, B. Perrin, E. J. Hinch, D. Salin, and J. P. Hulin, Phys. Fluids 19, 125105 (2007).

[24] R. P. Schwarzenbach, P. M. Gschwend, and D. M. Imboden, Environmental Organic Chemistry, 2nd ed. (John Wiley \& Sons, Hoboken, NJ, 2003).

[25] W. M. Haynes, in CRC Handbook of Chemistry and Physics, edited by W. M. Haynes, 91st (internet version) edition (CRC Press/Taylor and Francis, Boca Raton, FL, 2011).

[26] T. Séon, J. P. Hulin, D. Salin, B. Perrin, and E. J. Hinch, Phys. Fluids 18, 041701 (2006).

[27] B. C. Kneller, S. J. Bennett, and W. D. McCaffrey, J. Geophys. Res. 104, 5381 (1999).

[28] D. Moxey and D. Barkley, PNAS 107, 8091 (2010).

[29] P. Hazel, J. Fluid Mech. 51, 39 (1972). 